\title{
Forum
}

\section{A Decimal System of Celestial Navigation Based on the Second}

\author{
from K. K. White
}

I. INTRODUCTION. The purpose of this note is to outline a system of celestial navigation which is both decimal and coherent. In a decimal system there can only be one unit, with its decimal multiples and submultiples, for measuring any quantity. It follows that a decimal system of celestial navigation requires one unit for measuring time and one unit for measuring arc. In a coherent system these units must be related by a factor of one.

2. MEASURING TIME. The most sensible choice of conventional unit for measuring time is the second. It is a precisely defined unit (SI) ${ }^{\prime}$ to which all the other units of time are related and, if any other unit were chosen such as the minute, hour or day, it would have to be related to the second by factors of $1 / 60,1 / 3600$ or $1 / 86400$, making a decimal and coherent system unworkable.

There being 86400 seconds (s) in the daily G.M.T. time cycle, a digital chronometer recording in seconds would record from $I$ to $86400 \mathrm{~s}$, zero and start a new day. Since the second is a small unit, it will be convenient for certain purposes in navigation to bring into use the decimal multiple of the second, the kilosecond (ks) which is one thousand seconds.

3. MEASURING ARC. Picture, if you will, the hand of a large clock mounted at the centre of the Earth on the plane of the equator. With some assistance from the denizens of the nether regions the hand is manoeuvred to point in the direction of the international date line (IDL) at midnight G.M.T. when the clock is started. During the course of the day the hand roughly follows the Sun in its apparent rotation round the Earth and at the close of the day, or $86400 \mathrm{~s}$ later, the hand is again in line with the IDL.

At the end of the first second, the hand will have described an angle with the line joining the centre of the Earth to the IDL at the equator. This angle may be called the 'second of angle' and given the symbol $s_{A}$ to distinguish it from the second of time, $s$. Every subsequent second an additional $s_{A}$ will have been described until, at the end of the day, there will be 86400 of such units of angle, completing the circle. The $s_{A}$ is thus $1 / 86400$ of a circle. It should not be confused with the conventional second of arc (") which is a circle divided by $360 \times 60 \times 60$ or 1296000 . For purposes of comparison $1 s_{A}=\frac{1}{4}$ minute of arc.

The $s_{A}$, then, provides a simple unit for measuring arc which is consistent with a decimal and coherent system of celestial navigation.

4. LONGITUDE, LATITUDE AND DECLINATION. In a decimal system there can only be one unit of angle. The way to measure longitude, latitude and declination in $s_{A}$ is best illustrated in Figs $I$ and 2 . In each case there is only one base line and a progression in $s_{A}$ in one direction only. This avoids the confusion and dangers of error inherent in the traditional system. 


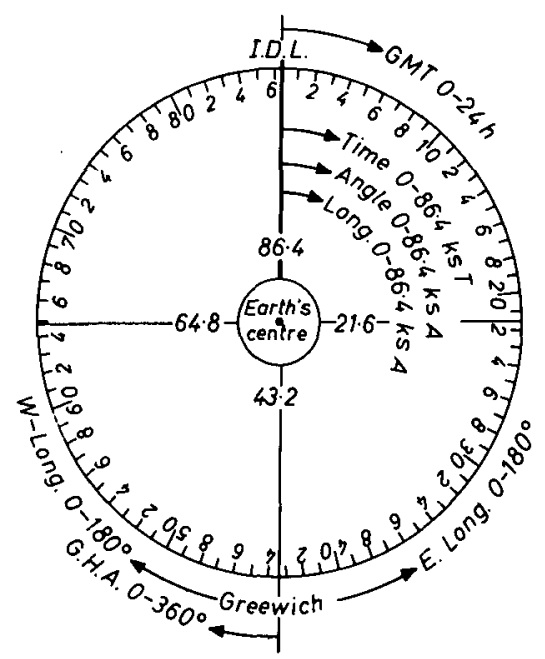

Fig. I. Time, angle and longitude

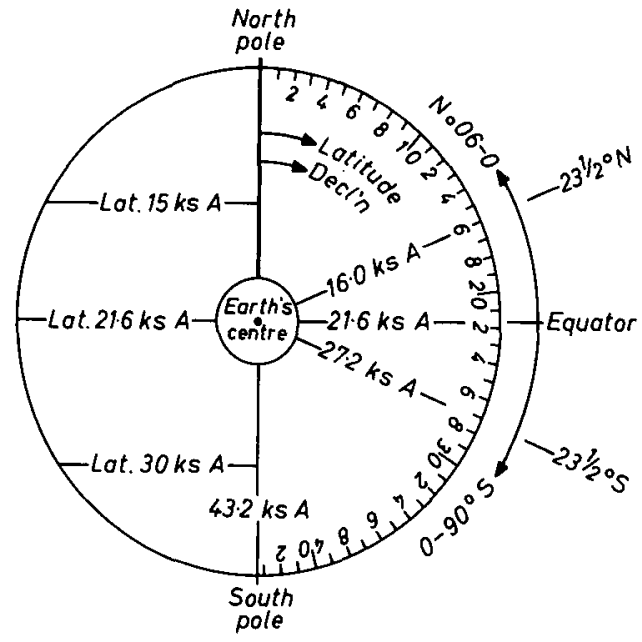

Fig. 2. Latitude and declination

Note: (1) Graduations are in kiloseconds of angle $\left(\mathrm{ks}_{\mathrm{A}}\right), \mathrm{s}_{\mathrm{A}}$ being too small to show on the drawing. (2) The IDL is assumed to lie along the whole length of longitude I $80^{\circ}$ E. or W. (3) Traditional systems are shown outside the circles

5. THE SUN'S MERIDIAN AND DECLINATION. If the Sun's apparent rotation round the Earth were entirely regular and its daily cycle were completed every $86400 \mathrm{~s}$ exactly, the navigator would not need an almanac to tell him its position at any time of the day. For instance, at $20000 \mathrm{~s}$ it would be directly above the meridian (longitude) of $20000 \mathrm{~s}_{\mathrm{A}}$. In fact, of course, when compared with the regular cycle of the chronometer, the Sun appears to start the day early or late in relation to the IDL and to gain or lose time during the day depending on the time of year. Therefore, the navigator needs an almanac to tell him the number of $s_{A}$ the Sun was early or late at the start of the G.M.T. day and the number of $s_{A}$ it will gain or lose each kilosecond during the day. With this information he can establish the Sun's meridian at any time by simple arithmetic.

Similar information about the Sun's declination in $s_{A}$ at $00000 \mathrm{~s}$ and the number of $\mathrm{s}_{\mathrm{A}} / \mathrm{ks}$ it increases or decreases during the day, enables the Sun's declination to be calculated for any second of the day.

6. THE LOCAL ANGLE OF THE SUN. The navigator compares his DR longitude with the Sun's meridian in $s_{A}$. To establish the local angle of the Sun he has only to subtract the smaller figure from the larger.

7. CALCULATION OF LATITUDE. Where TSA represents the true (corrected) angle in $s_{A}$ of the Sun at the observer's noon, his latitude is obtained by subtracting the TSA from $21600 \mathrm{~s}_{\mathrm{A}}$ (the equivalent of $90^{\circ}$ ). The result is then subtracted from the declination in $\mathrm{s}_{\mathrm{A}}$ when the Sun is to the south of him or added when it is to the north.

8. OTHER CELESTIAL Bodies. Measurement and calculations in $s_{A}$ of the meridians and declinations of the stars and other celestial bodies, as well as the observer's relationship to them, follow the same lines as those relating to the Sun.

9. FINDING A POSITION LINE. The change in units allows calculations to be simplified without radical change in accepted methods of establishing position lines. 
10. THE COMPASS AND SEXTANT. A compass card graduated in $\mathrm{ks}_{\mathrm{A}}$ would resemble that illustrated in Fig. I. North would take the place of the IDL while east, south and west would be indicated at $21.6 ; 43.2$ and $64.8 \mathrm{ks}_{\mathrm{A}}$ respectively. The arc of the sextant would be graduated to $30 \mathrm{ks}_{\mathrm{A}}$.

I I. AN ALTERNATIVE. The system which has been outlined could alternatively be based on a natural decimal division of the day and the circle into roo0 millidays (md) instead of 86400 seconds. Developments, in the application of piezo-electricity, make it possible to give consideration to such a radical departure from traditional ways of measuring time.

12. CONCLUSION. If, like other scientific disciplines, celestial navigation adopted the second as the unit of time, a system such as that described is, in the writer's view, the only conceivable one which is both decimal and coherent.

\section{REFERENCES}

1 SI The International Systern of Units (1970) HMSO.

2 Gardner, A. C. (1973). Navigation. English Universities Press.

\section{Alton B Moody comments}

The decimal system suggested by $K$. K. White is the latest of a number of systems suggested over the years. While any decimal system, with its simple relationships, has an obvious appeal, there seems to be little to recommend a system based upon the second of time, with its unnatural relationship to the circle.

Even simple suggestions such as measuring longitude, like Greenwich hour angle, in one direction through 360 degrees, and latitude and declination from pole to pole, through 180 degrees, have met with little favour among navigators. A suggestion that the number of degrees in a circle be changed to 400 to provide a decimal system of sorts and that a corresponding change be made in the number of hours in the day, while providing a relatively simple system with obvious advantages, has fallen on deaf ears. There is not much appeal to a circle graduated to 86400 units.

Not only is mental inertia involved, but natural relationships such as that between a minute of arc and a nautical mile and that between 15 degrees of longitude and an hour of time have, in my view, too much practical merit to be discarded in favour of any nebulous advantages that might be associated with the suggested method.

\section{Speed Control: A Useful Tool of VTS}

\section{Capt. C. W. Koburger, Jr. USCGR (Retd)}

I. INTRODUCTION. This paper discusses some of the implications of speed control in the vessel traffic picture, emphasizing how speed relates to other safety factors so that control can be utilized with understanding and sensitivity. Speed control can seldom if ever be examined in isolation. The structuring of vessel traffic schemes implies a need for some form of speed control as an integral part of almost all those schemes. One technique reinforces the other. Speed limits become extremely critical at the lower end of the speed scale, the higher the traffic density, the more critical the limits. Again, the requirement for enforcement becomes clear. No over-emphasis of these operational 